\title{
Clinical pathway for an efficient wound care center
}

\author{
Yu-Tsung Chen ${ }^{1,2}$, Chang-Cheng Chang ${ }^{3,4}$ \\ ${ }^{1}$ Department of Dermatology, Shuang Ho Hospital, Taipei Medical University, Taipei 110, Taipei, China. \\ ${ }^{2}$ School of Public Health, College of Public Health and Nutrition, Taipei Medical University, Taipei 110, Taipei, China. \\ ${ }^{3}$ Aesthetic Medical Center, China Medical University Hospital, Taichung 404, Taipei, China. \\ ${ }^{4}$ School of Medicine, China Medical University, Taichung 404, Taipei, China.
}

Correspondence to: Prof. Chang-Cheng Chang, Aesthetic Medical Center, China Medical University Hospital, Taichung 404, Taipei, China.

E-mail: changcc1975@gmail.com.

How to cite this article: Chen YT, Chang CC. Clinical pathway for an efficient wound care center. Plast Aesthet Res 2016;3:375-7.

Article history: Received: 30-11-2015 Accepted: 30-12-2015 Published: 20-12-2016

Dr. Chang-Cheng Chang is a well-known microvascular reconstructive and aesthetic plastic surgeon. He is the
founder of both Wound Care Center and Aesthetic Medical Center in Chang Gung Memorial Hospital, Chia Yi, in
2010. Dr. Chang had focused his studies on wound care and aesthetic laser medicine, and had published several
papers which has proven to help increase treatment outcome in the past five years. He also works closely with the
Wound Care Center team which further comprises of plastic surgeons, cardiovascular and metabolic physicians,
rehabilitation specialists, social workers, nursing specialists and coordinators. Until present, he has published 22
peer-review scientific articles and authored one book chapter. Dr. Chang has also given numerous lectures at
various international societies and centers and received national honorable awards. He is now serving as the head
in Aesthetic Medical Center, China Medical University Hospital, Taichung, Taipei, China.

\section{INTRODUCTION}

Chronic wound significantly influence patients' quality of life, but wound care procedures can vary between hospitals. Wound management difficulties can be attributed to lack of standardized protocol, wellintegrated multidisciplinary team, and specific wound care unit. An establishment of wound care center can help to solve above difficulties with providing wound management service.

The efficient 3-step wound management pathway has the advantage that all involved specialists from different departments in the hospital can keep tracing the patients' condition in the same pre-existing unit of the healthcare system. In addition to improving the quality of wound care, the establishment of a wound care center will not only improve patients' pain and activities of daily life, but also achieve biopsychosocial healthcare, an approach believed to have positive effects on reducing costs and relieving the burden on the healthcare system.

\section{WOUND MANAGEMENT DIFFICULTIES}

The goals of wound management service are to treat 


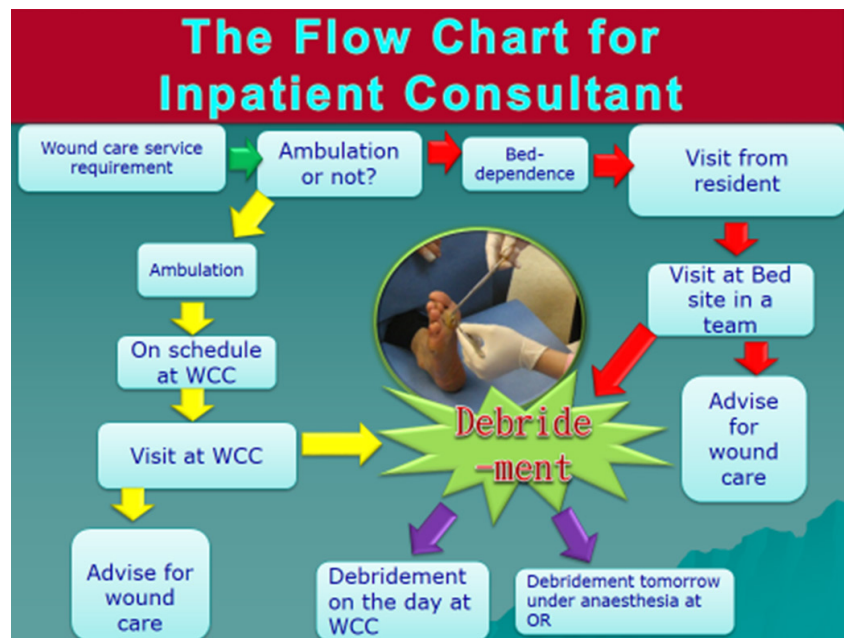

Figure 1: The conceptual framework for wound management service contains three steps, including patient entry/on-site debridement, wound re-evaluation, and individual wound bed preparation. If the ambulation is possible, we will visit the patient at WCC and do the wound management service including debridement or wound care advice. If not, we will go to bed side for visit. OR: operating room; WCC: wound care center

chronic wounds through the stages of healing, reduce infections or complications, and prevent future chronic wounds as well as restore the functional activities of daily life. Although the benefits of wound care service and multidisciplinary teams care have been well discussed, ${ }^{[1-3]}$ there is a practicing gap about the establishment of an efficient referral system. Patients are often confused regarding whom to see for wound care, and simply receive wound treatment by the individual specialists they visit, in which the specialists may not actively track wounds unless they are consulted again. And healthcare providers (plastic specialists, dermal specialists, general practitioners, nurses) also found it difficult to manage wound care across disparate levels of departments. Furthermore, a lack of organization and specific space may postpone the accurate screening and debridement on-site and ontime. Therefore, the establishment of clinical pathway for wound management service/pathway is crucial.

\section{WOUND MANAGEMENT PATHWAY}

The wound management pathway consists of three steps. The first step is patient entry and onsite immediate wound debridement, which indicates the patient referral process where wound center clients were referred from outpatient clinics and inward consultants. The wound care service is initiated once the physician from any other departments considered wound care service necessary. The patient is scheduled for a visit to the wound care center by the coordinator, and the appointed doctor can meet the patient and family on the arranged day to discuss the wound condition with them. Before the wound management pathway was established, wound inpatients were referred to different specialized departments such as dermatology, surgery, and internal medicine, where wound management care was limited to that department's particular function. And the wound consultant by plastic surgeon usually took a few days even in urgent cases. In wound management pathway, without an uncertain waiting period, senior consultants can approach patients in person with professional wound assessments and perform immediate debridement on-site as needed [Figure 1]. The therapeutic process lags and can be delayed because the chronic wound may need a series of debridement and regular follow-up. The coordinator (nursing staff) also assisted the consultant in scheduling follow-up appointments at the center as clinically needed. The wound care center provides not only one-time wound care, but also a complete program of care including further education and prevention for patients and families.

After being referred and completing the first visit and debridement, the patients proceeded to step two, wound re-evaluation, including neurologic evaluation, vascular evaluation (e.g. Ankle-Brachial Index), and the TIME concept (Tissue, Infection/Inflammation, Moisture imbalance and Edge of wound). ${ }^{[4]}$ Wound size is measured by the longest length and width of the wound. All individual patient wound profiles are organized into our data bank, which can be analyzed for wound care efficacy and follow-up.

The third step includes setting up the individual wound care plan based on the results of wound re-evaluation in step two. Emphasis is placed on the importance of wound bed preparation (using TIME), early referral between members of the multidisciplinary team, such as vascular team, hyperbaric oxygenation therapy, further education and prevention. Offloading must therefore be addressed in a timely manner.

\section{THE EFFICIENT REFERRAL SYSTEM FOR WOUND CARE CENTER}

Multiple studies have supported the efficacy of protocolbased treatmentfor pressure ulcers, venous stasis ulcers, and diabetic foot ulcers. ${ }^{[5-7]}$ Edwards et al. ${ }^{\left[{ }^{[8]}\right.}$ confirmed that the implementation of evidence-based protocols for wound assessment and treatment was significantly related to improve healing outcomes. The principle of wound care protocol is easy to comprehend, but the execution of the clinical efficient wound management service is complicated and needs a standardized and well-integrated multidisciplinary team with specific capacity. The main reason for difficulty in wound care is the poor referral system, which may be attributed to 
Table 1: Comparison between wound management service and conventional system

\begin{tabular}{lccc}
\hline & Conventional system & Three-step wound management service \\
\hline Specific wound care space & - & +++ \\
On-time visit and on-site debridement & - & +++ \\
Integrated multidisciplinary team & + & +++ \\
Feasibility for regular follow-up & + & +++ & + \\
\hline
\end{tabular}

The three-step wound management service has the advantage of specific wound care unit space, scheduled visit and on-site debridement, integration of multidisciplinary team, and regular following-up

the inability of the physician to identify and diagnose the wound, patient refusal to see the doctor, patient refusal to be referred to a wound care center, and the absence of a highly efficient wound care center. The lack of education and routine training for professional wound care and the huge variety of wound care products, procedures, and treatments pose a potential problem for the implementation of wound care as well.

The major and significant improvement by wound care center mainly relies on the referral system for patients, which decreases the possibility of avoiding delay of early debridement. Comparing to conventional wound care system, the three-step wound management service has the advantage of specific wound care unit space, scheduled visit and on-site debridement, integration of multidisciplinary team, and regular following-up [Table 1]. When wound care center had not been established yet, the assessor for wound consultation was usually the training physician, rather than consultants (surgeon), as they were already busy with other daily practice activities. Hospitalized patients may wait until the night for a visit as they waited until the assessor finished the daily routines. Instead of visiting the patient personally, the senior consultant sometimes assessed the wounds from only photos taken by the training physician. Then, the senior consultant still needed to arrange the next scheduled operation room for wound management (debridement, amputation or flap); this process usually took a few days even in urgent cases. Scheduling was based around the surgeon's activities rather than the patients' requirement. The delay of debridement could have caused processing or even deterioration of the wound. With the integration of the efficient standardized multidisciplinary teams in a specific wound care center, patients can schedule appointments instead of waiting indefinitely for care. Staff can approach patients in person, administer an adequate wound assessment, and perform on-time debridement. Further wound condition follow-up, education, and prevention are also continually provided.

\section{Financial support and sponsorship}

None.

\section{Conflicts of interest}

There are no conflicts of interest.

\section{Patient consent}

No patient involved.

\section{Ethics approval}

This kind of paper doesn't need ethical approval.

\section{REFERENCES}

1. Doan-Johnson S. The growing influence of wound care teams. $A d v$ Wound Care 1998;11:54.

2. Steed DL, Edington H, Moosa HH, Webster MW. Organization and development of a university multidisciplinary wound care clinic. Surgery 1993;114:775-8; discussion 778-9.

3. Jaramillo O, Elizondo J, Jones P, Cordero J, Wang J. Practical guidelines for developing a hospital-based wound and ostomy clinic. Wounds 1997;9:94-102.

4. Chin GA, Schultz G, Stacey M. Principles of wound bed preparation and their application to the treatment of chronic wounds. Wound Practice Res 2004;11:171-4.

5. Kjaer ML, Sorensen LT, Karlsmark T, Mainz J, Gottrup F. Evaluation of the quality of venous leg ulcer care given in a multidisciplinary specialist centre. J Wound Care 2005;14:145-50.

6. Hsu CR, Chang CC, Chen YT, Lin WN, Chen MY. Organization of wound healing services: the impact on lowering the diabetes foot amputation rate in a ten-year review and the importance of early debridement. Diabetes Res Clin Prac 2015;109:77-84.

7. Chen YT, Chang CC, Shen JH, Lin WN, Chen MY. Demonstrating a conceptual framework to provide efficient wound management service for a wound care center in a tertiary hospital. Medicine (Baltimore) 2015;94:e1962.

8. Edwards H, Finlayson K, Courtney M, Graves N, Gibb M, Parker C. Health service pathways for patients with chronic leg ulcers: identifying effective pathways for facilitation of evidence based wound care. BMC Health Serv Res 2013;13:86. 